\title{
Lipodisqs for eukaryote lipidomics with retention of viability: sensitivity and resistance to Leucobacter infection linked to C.elegans cuticle composition
}

Juan F. Bada Juarez ${ }^{1}$, Delia O’Rourke ${ }^{1}$, Peter J. Judge ${ }^{1}$, Li C. Liư ${ }^{1}$, Jonathan Hodgkin ${ }^{1 *}$ and Anthony Watts ${ }^{1 *}$

${ }^{1}$ Department of Biochemistry, University of Oxford, South Parks Road, OX1 3QU, Oxford

*corresponding authors: anthony.watts@bioch.ox.ac.uk, jonathan.hodgkin@bioch.ox.ac.uk

\section{Abstract}

Lipodisq $^{\text {TM }}$ nanoparticles have been used to extract surface lipids from the cuticle of two strains (wild type, N2 and the bacterial-resistant strain, agmo-1) of the C. elegans nematode without loss of viability. The extracted lipid were characterized by thin layer chromatography and MALDI-TOF-MS. The lipid profiles differed between the two strains. The extracted lipids from the bacterial resistant strain, agmo-1, contained ether-linked (O-alkyl chain) lipids, in contrast to the wild type strain which contained exclusively ester (O-acyl) linked lipids. This observation is consistent with the loss of a functional alkylglycerol monooxygenase (AGMO) in the bacterial resistant strain agmo-1. The presence and abundance of other lipid species also differs between the wild-type N2 and agmo-1 nematodes, suggesting that the agmo-1 mutant strain attempts to compensate for the increase in ether-linked lipids by modulating other lipidsynthesis pathways. Together these differences not only affect the fragility of the cuticle and the buoyancy of the worm in aqueous buffer, but also interactions with surface-adhering bacteria. The much greater chemical stability of O-alkyl, hydrolysable linked lipids compared with non-hydrolysable O-acyl linked lipids, may be the origin of the bacterial resistance of the agmo-1 strain, providing a more resilient cuticle for the nematode. Additionally, we show that lipid extraction with a polymer of styrene and maleic acid (SMA) provides a viable route to lipidomics studies with minimal perturbation of the organism. 


\section{Introduction}

C. elegans has been a successful model organism for the study of both animal development and behaviour for more than 50 years ${ }^{1,2}$. The genome was the first from a multicellular organism to be sequenced ${ }^{3}$ and their simplicity makes them a perfect system for studies of gene expression, ageing, development and the nervous system ${ }^{4}$. The $C$. elegans 'exoskeleton' ${ }^{5}$, the cuticle, which forms a barrier between the external environment and the worm epidermis, is poorly characterized. The cuticle has an important role in C. elegans biology, as it maintains body shape while permitting locomotion and nutrition, and also offers protection against bacterial and fungal parasites ${ }^{6}$.

Previously, we described the isolation of two new Leucobacter bacterial pathogens of Caenorhabditis, discovered co-infecting a wild isolate collected in Cape Verde ${ }^{7}$. The interactions of these bacteria with $C$. elegans revealed unusual mechanisms of pathogenic attack: one pathogen, Verde1, traps swimming nematodes by sticking their tails together, resulting in the formation of "worm-star" aggregates, in which worms were killed and degraded; the other pathogen, Verde2, kills worms by a different mechanism associated with rectal infection. Both infections require the attachment of bacteria to the cuticle for their pathogenic effects ${ }^{7}$.

Genetic screens identified many distinct mutant worms that were resistant to lethal Leucobacter Verde2 infection. These mutants alter the chemical properties of the worm surface, preventing Verde2 attachment and also affecting movement, sensitivity to bleach and the binding of lectins. The Verde2-resistant mutants are however hypersensitive to Leucobacter Verde1 infection. Further genetic suppressor screens to reverse the lethal sensitivity of these mutants to Verde1 suggested a common mechanism involved in suppression of Verde1 infection, identified as the loss of the pathway required for the degradation of ether lipids. Many independent suppressor mutations were found in the gene encoding alkylglycerol monooxygenase (AGMO), a metabolic enzyme solely responsible for the degradation of etherlinked lipids 8,9 , or in enzymes required for the synthesis of its essential cofactor tetrahydrobiopterin ${ }^{8,10}$. This striking reciprocal sensitivity and resistance to Verde1 and Verde2 may reflect the fact that these pathogens were isolated as a co-infection of wild-type 
Caenorhabditis, suggesting that the worms may have developed a trade-off relationship between sensitivity and resistance to the two pathogens ${ }^{7}$.

The finding that loss of the ether lipid degradation pathway confers resilience to Verde1 infection, suggested that changes in the lipid content of the C. elegans cuticle are responsible for altering the susceptibility to the Verde1 and Verde2 infection mechanisms ${ }^{7}$. Here we complement the genetic analysis of $C$. elegans surface with a biochemical analysis of the cuticle lipidome, both in mutants that demonstrate resistance to Verde1 infection and in wild-type worms.

The $C$. elegans cuticle is secreted by the underlying epithelial cells and is regenerated entirely following moulting at four larval growth stages (L1-4). It is thought that the thickness of the cuticle, as well as its protein and lipid composition, change at every larval stage and may vary in response to the environment ${ }^{4,5}$. The cuticle consists of several layers, the outermost being the surface coat, which is covered by glycoproteins and has a net negative charge at neutral $\mathrm{pH}$, due to the presence of sugar-sulphate moieties ${ }^{6}$. This outermost layer is likely to play an important role in locomotion and may prevent the adhesion of microbes to the nematode surface $^{5}$. C. elegans srf mutants, with an altered carbohydrate epitope expression profile on the coat, have a weakened cuticle and modified bacterial adherence ${ }^{5}$. Other additional bus (Bacterially UnSwollen) mutants show similar phenotypes, including modified surface lectin binding ${ }^{11}$.

Below the hydrophilic surface coat, the epicuticle contains both glycoproteins and lipids, including non-polar species such as steroids (particularly cholesterol), polar lipids such as PC and PE and some glycolipids, which appear to have important roles in the immune response $\mathrm{e}^{6,12}$. With the exception of the epicuticle and the surface coat, the cuticle is predominantly made of collagen and its derivatives (cross-linked and collagen-like molecules) ${ }^{13}$. The epicuticle of the parasitic nematode Trichinella spiralis, is mainly composed of PC, PE and PG and has lipids arranged in an inside-out cylindrical configuration, in which the hydrophilic headgroups point inwards, exposing the hydrophobic tails to the rest of the cuticle ${ }^{14}$. 
Lipid mobility in the cuticles of several nematode species is restricted compared to a typical plasma membrane ${ }^{4,15}$. The presence of lipids in the epicuticle of other nematodes has been assessed by fluorescent probes and lipase treatment ${ }^{4,5,16,17}$ and the flexibility and resistance to chemical and physical stresses have been attributed to the presence of some waxes and/or glycolipids (ascarosides ${ }^{17}$ ). Conversely, proteins in the epicuticle have been shown to diffuse quickly, which is attributed both to weak ionic interactions with lipids and also due to their distribution in membrane domains containing neutral lipids, in which they can diffuse freely $y^{4,15}$.

We set out to identify the components of the nematode surface in order to understand how defects in lipid metabolism change the surface of $C$. elegans and thereby alter patterns of bacterial adherence. We focused on differences between wild-type N2 worms and the most frequently identified Verde1 suppressor mutant, agmo-1, which lacks alkylglycerol monooxygenase $(\mathrm{AGMO})^{8,9}$. By disrupting the metabolic pathways responsible for lipid synthesis $^{9}$, loss of the enzyme might alter the sensitivity towards bacterial pathogens through a destabilization of the cuticle. While the total lipidome of $C$. elegans has been partially analysed using imaging, genetic and biochemical techniques ${ }^{16,18-20}$, the characterization the of $C$. elegans surface has been limited ${ }^{6}$, owing to the difficulty of selectively extracting lipids from the cuticle, without contamination from other cellular membranes in the worm.

SMA (a hydrolysed co-polymer of styrene and maleic anhydride) has recently been shown to be able to simultaneously extract both lipids and proteins from biological membranes. The polymer extracts lipids non-selectively, preserving protein structure and activity ${ }^{21-25}$. The resulting nanoparticles (commonly termed Lipodisq or SMALPS) have also been used for structural characterization by cryo-EM ${ }^{26,27}$ and X-ray crystallography ${ }^{28}$. Several membrane proteins including ion channels ${ }^{29}$ and $\mathrm{GPCRs}^{30,31}$ have been studied and characterized from different organisms ${ }^{32-35}$ and some proteomics and lipidomics experiments have been performed using SMALPs by hydrogen-deuterium exchange mass spectrometry and LC$\mathrm{MS} / \mathrm{MS}^{36,37}$.

Here, SMA polymer is used to extract the lipids present in the nematode cuticle of $C$. elegans wild-type (N2) and agmo-1 strains. We characterise the surface lipids from the wild-type and mutant strains of $C$. elegans using MALDI-TOF-MS. Our aim is to identify and understand the 
molecular differences in cuticle composition and structure, which underpin the different phenotypes of the wild-type and surface mutant nematodes. 
2. Material and methods

\subsection{C. elegans culture}

The N2 (wild-type) and agmo-1 (strain CB7014, genotype agmo-1(e3016)) nematodes were grown on nematode growth media plates ${ }^{2}$. The generations were synchronized in order to obtain a homogeneous population prior to SMA polymer addition.

\subsection{Formation of Lipodisq nanoparticles}

SMA polymer containing a molar ratio of styrene:maleic acid of 3:1 was supplied by Malvern Cosmeceutics Ltd (Malvern, UK). Synchronised populations of worms were harvested by washing the media plates with $10 \mathrm{mM}$ Tris $5 \mathrm{mM} \mathrm{NaCl} \mathrm{pH} 8$ followed by three rounds of centrifugation $\left(2500 \mathrm{~g}, 2\right.$ minutes, $4^{\circ} \mathrm{C}$ ). An SMA solution ( $25 \% \mathrm{w} / \mathrm{v}$ in $50 \mathrm{mM}$ Tris buffer $\mathrm{pH} 8$ ) was added to the washed worms at a final concentration of $12.5 \% \mathrm{v} / \mathrm{v}$ in $50 \mathrm{mM}$ Tris buffer $\mathrm{pH}$ 8 and incubated for 30 minutes at $30-40^{\circ} \mathrm{C}$. The sample was centrifuged, and the supernatant was kept for further analysis.

\subsection{Dynamic light scattering measurement}

The size of the Lipodisq nanoparticles, generated by lipid extraction by SMA, was measured by Dynamic Light Scattering at $633 \mathrm{~nm}$ using a Malvern Zetasizer Nano S instrument with a disposable cuvette. Data were processed using Malvern Zetasizer software.

\subsection{Viability test of worms after SMA treatment}

Worms were deposited onto a freshly seeded OP50 NGM plate and left at room temperature. The recovery was assessed from the percentage of worms that crawled across the OP50 making visible tracks. Plates were observed under a dissecting microscope after 1, 2, 4 and 24 hours, and were compared to those with nematodes that had not been exposed to SMA.

\subsection{Lipids extraction from Lipodisq nanoparticles}

Lipids were extracted from the Lipodisq samples according to the method of Bligh and Dyer ${ }^{38}$. Briefly, $200 \mu \mathrm{L}$ Lipodisq-containing sample was mixed vigorously for $1 \mathrm{~h}$, with $2 \mathrm{~mL}$ 
chloroform/methanol (2:1 v/v). Phase separation was achieved by adding $1 \mathrm{~mL}$ of $\mathrm{dd}_{2} \mathrm{O}$ water. The extract was left for 10 minutes at room temperature and then centrifuged (1,000 g, $10 \mathrm{~min}$ ) and the lower phase was retrieved washed twice with a methanol/water mix (1:1 v/v) and dried under a stream of $\mathrm{N}_{2}$ gas.

\subsection{Matrix-assisted laser desorption ionization mass spectrometry}

Water and DHB matrix were spotted onto a MALDI plate (MTP 384 Polished Steel TF Targets) and allowed to dry at room temperature. Lipids were resuspended in chloroform/methanol mixture $(2: 1 \mathrm{v} / \mathrm{v})$, deposited above the dried matrix and allowed to dry at room temperature. MALDI TOF mass spectra were acquired using a Bruker Ultraflex TOF/TOF operating in linear positive ion mode. MALDI laser intensity was selected to provide optimal intensity and resolution of acquired mass spectra. External calibration was performed using protein standards and some lipids standards, which were analysed under identical conditions. The identification of lipid was performed using LIPID MAPS online searching tools ${ }^{39}$ and data analysis was performed using the Thermo XCalibur (ThermoFisher) processing software and plotted in Prism (GraphPad software). 


\section{Results}

To determine the cuticle lipid composition of both wild-type (N2) and mutant (agmo-1) C. elegans, nematodes were incubated with SMA polymer. Both the time of incubation and temperature were adjusted to ensure that both wild-type and mutant worms recovered after the SMA treatment as shown in Figure 1. The best conditions for the SMA extraction of the nematode surface were found to be a temperature of between $30^{\circ} \mathrm{C}$ and $40^{\circ} \mathrm{C}$ and an incubation time of 30 to 60 minutes.

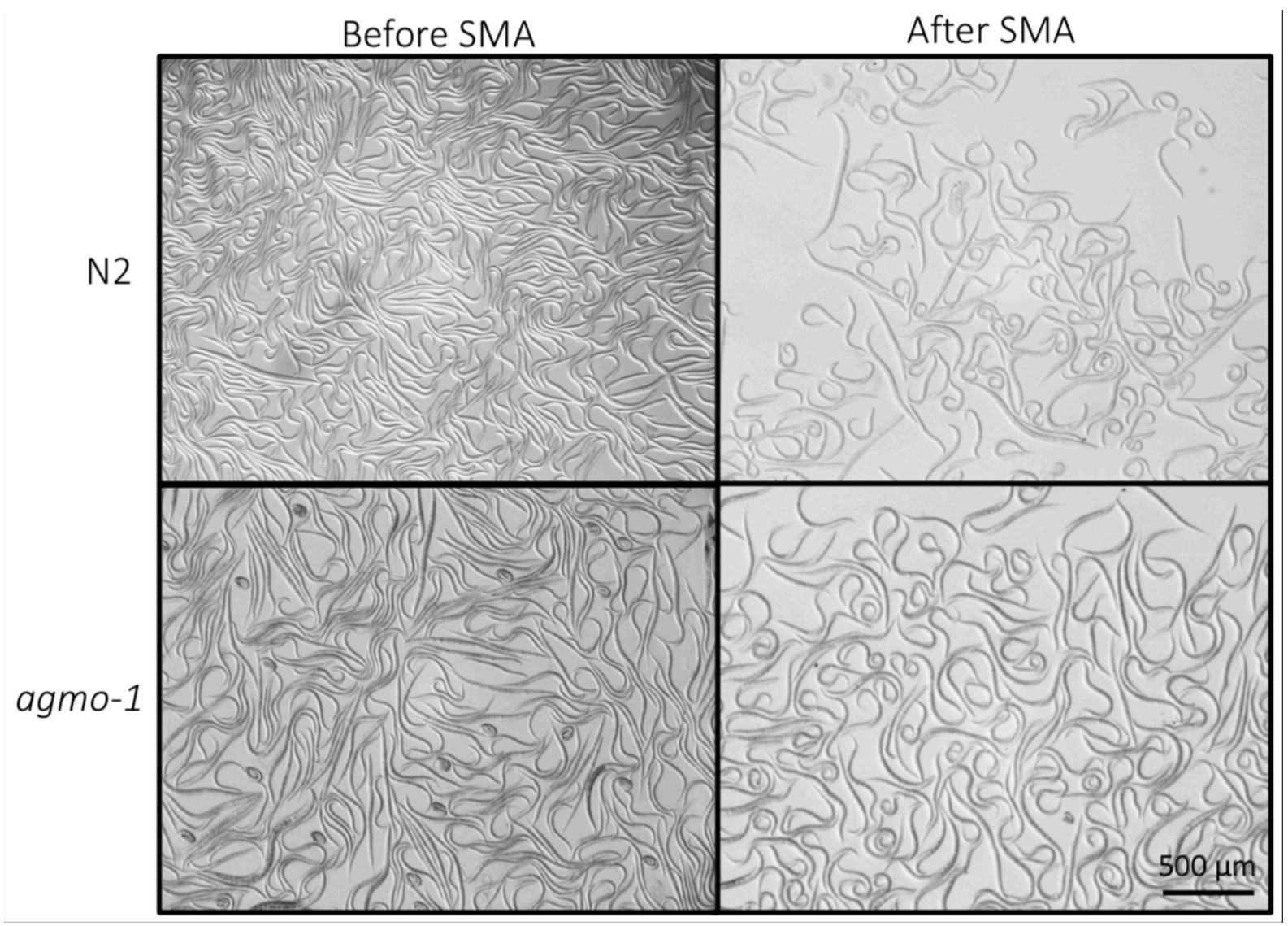

Figure 1 Synchronised worms before and after addition of SMA polymer. The viability and integrity of the worms was not affected as shown here.

After incubation, the SMA-worm suspension was centrifuged, the supernatant was collected and DLS measurements were performed to verify Lipodisq nanoparticle formation (Figure 2; Table 1). DLS data show that Lipodisqs were present in the supernatant (peak at 4-6 $\mathrm{nm}$ ), 
although some excess SMA was still present in the N2 strain sample (identified by the peak at $1.3 \mathrm{~nm}$. The agmo-1 mutant strain did not contain any excess SMA, despite the fact that the same number of worms was used to perform this experiment as for the wild type. A viability test showed that more than $95 \%$ of the worms survived the SMA incubation (data not shown).

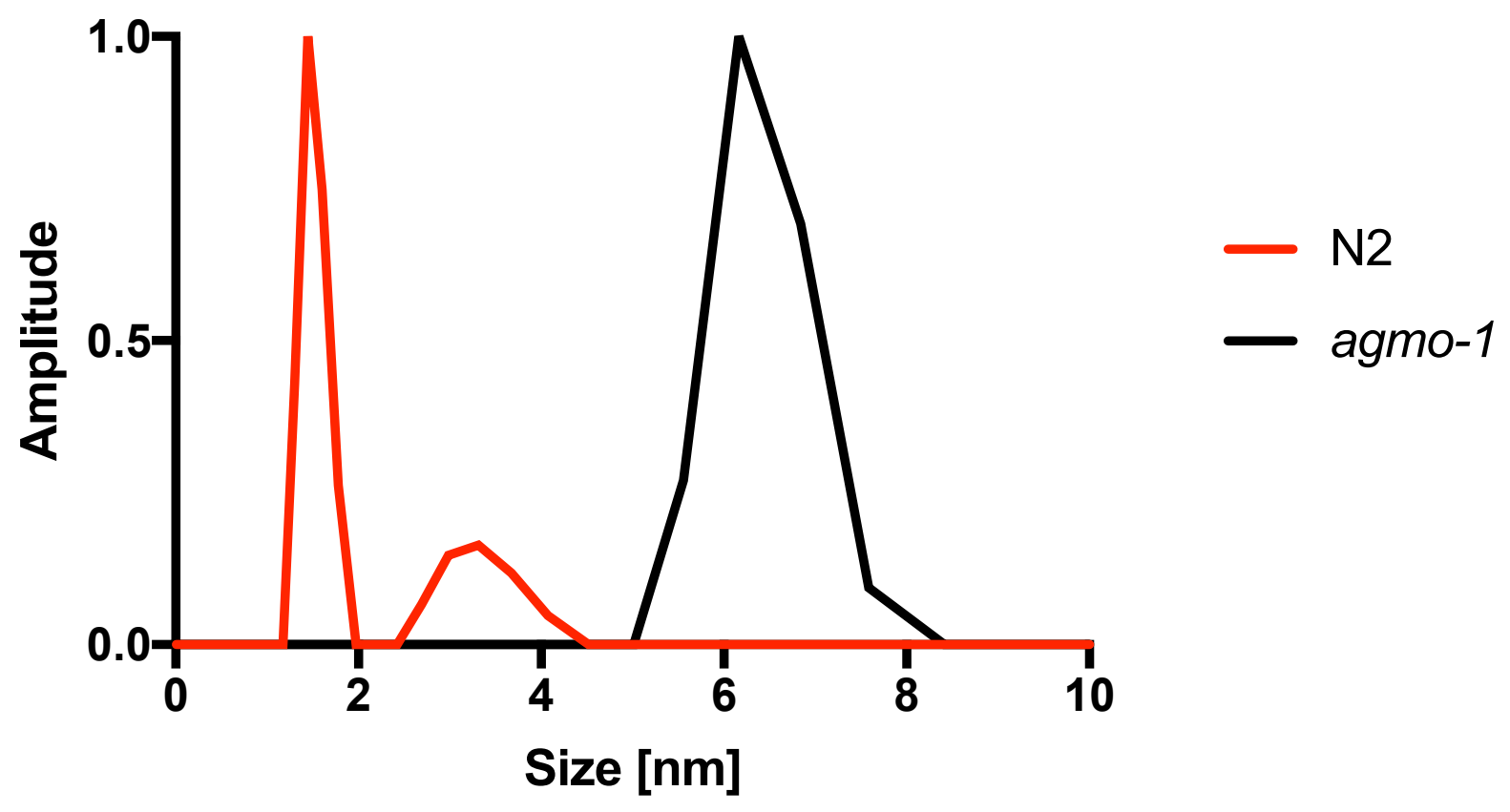

Figure 2 Dynamic light scattering experiment showing the different sizes obtained for both strains after SMA addition.

Table 1 DLS data for the SMA nanoparticles from the wild-type (N2) and the mutant strain (agmo-1)

\begin{tabular}{|l|c|c|}
\hline \multicolumn{1}{|c|}{ Peak } & N2 & agmo-1 \\
\hline SMA polymer & $1.5 \pm 0.1 \mathrm{~nm}$ & - \\
\hline Lipodisq nanoparticles & $3.3 \pm 0.4 \mathrm{~nm}$ & $6.4 \pm 0.4 \mathrm{~nm}$ \\
\hline
\end{tabular}

After the DLS analysis, the lipid composition of the Lipodisq nanoparticles from both nematode strains were investigated. Lipids were extracted by following the method of Bligh and Dyer ${ }^{38}$ and were analysed by thin layer chromatography (TLC) (Figure SI.1), as described previously ${ }^{29,36}$. Lipid headgroups were identified by their retention factor $\left(R_{f}\right)$ values (Table 2$)$. Ceramides, commonly found in the skin of mammals ${ }^{40}$ in which they appear to play a role in the organisation of other lipids ${ }^{41}$, are found in both wild type and mutant worms, and their appearance is consistent with the slow lipid diffusion rates previously reported in the cuticle ${ }^{4,15,42}$. Also present in both strains were cerebrosides $(\mathrm{Cb})$ and lyso-phosphatidylcholine (LPC). For the N2 strain, lipids such as phosphoethanolamine (PE), lyso-phosphatydilglycerol 
(LPG), sphingosine/sphingomyelin (SM) and lyso-phosphatydilserine (LPS) were found, whereas in the mutant agmo-1, phosphatidyl-N-monomethylethanolamine (PMME), cardiolipin (CL) and PG were found. Also, some lipid species are not able to migrate in the chosen solvent mixture, and this might indicate the presence of highly charged species as speculated by Blaxter and colleagues 6 .

Table 2 The different lipids identified (with their retention factors in brackets) by TLC and compared to the literature. ND, unidentified lipids.

\begin{tabular}{|r|c|c|}
\hline N2 & agmo-1 & N2 from Blaxter \\
\hline PE (0.79) & PMME (0.71) & PI (0.09) \\
\hline LPG (0.54) & CL (0.67) & ND (0.24) \\
\hline SM (0.28) & PG (0.6) & PC (0.38) \\
\hline LPS (0.18) & - & PE (0.5) \\
\hline CE (1) & ND (0.61) \\
\hline Cb (0.94) & Sterol (0.9) \\
\hline LPC (0.22) & ND (1) \\
\hline
\end{tabular}

\begin{tabular}{|c|c|}
\hline Glycerophosphocholines & PC (LPC for lyso species) \\
\hline Glycerophosphoethanolamines & PE (LPE for lyso species) \\
\hline Glycerophosphoserines & PS (LPS for lyso species) \\
\hline Glycerophosphoglycerols & PG (LPG for lyso species) \\
\hline Glycerophosphates & PA (LPA for lyso species) \\
\hline Glycerophosphoinositols & PI (LPI for lyso species) \\
\hline Glycerophosphoglycerophosphoglycerols (Cardiolipins) & CL \\
\hline Monoradylglycerolipids & MG \\
\hline Diradylglycerolipids & DG \\
\hline Triradylglycerolipids & TG \\
\hline Digalactosyldiacylgylcerols & DGDG \\
\hline Sulfoquinovosyldiacylglycerols & SQDG \\
\hline Ceramides/Sphingoid bases & Cer/Sph \\
\hline Hexosyl ceramides & HexCer \\
\hline Mannosyl-PI-ceramides & MIPC \\
\hline Mannosyl-di-PI-ceramides & M(IP) \\
\hline Cactosyl ceramides \\
\hline Sulfatides & LacCer \\
\hline Wax esters & SHexCer \\
\hline Cholesteryl esters & WE \\
\hline Fatty acids & CE \\
\hline FA \\
\hline Lyso-phosphatidylinositol-monophosphate & LPIP \\
\hline
\end{tabular}

TLC is not able to distinguish between ester- and ether-linked lipids with the same headgroup and MALDI-TOF MS was therefore used to assist in the identification of some of the lipids present. As some of these lipids have a mass below $1 \mathrm{kDa}$, the instrument was calibrated using low molecular weight protein standards in addition to lipids such as DMPC, POPC, DOPC and POPG. Moreover, as the $500 \mathrm{Da}$ to 1000 Da mass range is difficult to study due to the noise peaks produced by the matrix, a control experiment including the recording of mass spectra of matrix-only spots was performed (data not shown) and the peaks of this matrix-only experiment were subtracted from the Lipodisq sample experiments. Another control was performed which includes the removal of the peaks coming the OP50 bacterial source lipids extracted using SMA. Matrix-only peaks were subtracted from the mass spectra of N2 and agmo-1. The mass spectra for the N2 and agmo-1 after removal of both the matrix and OP50 lipids peaks are shown in Figure 4. 


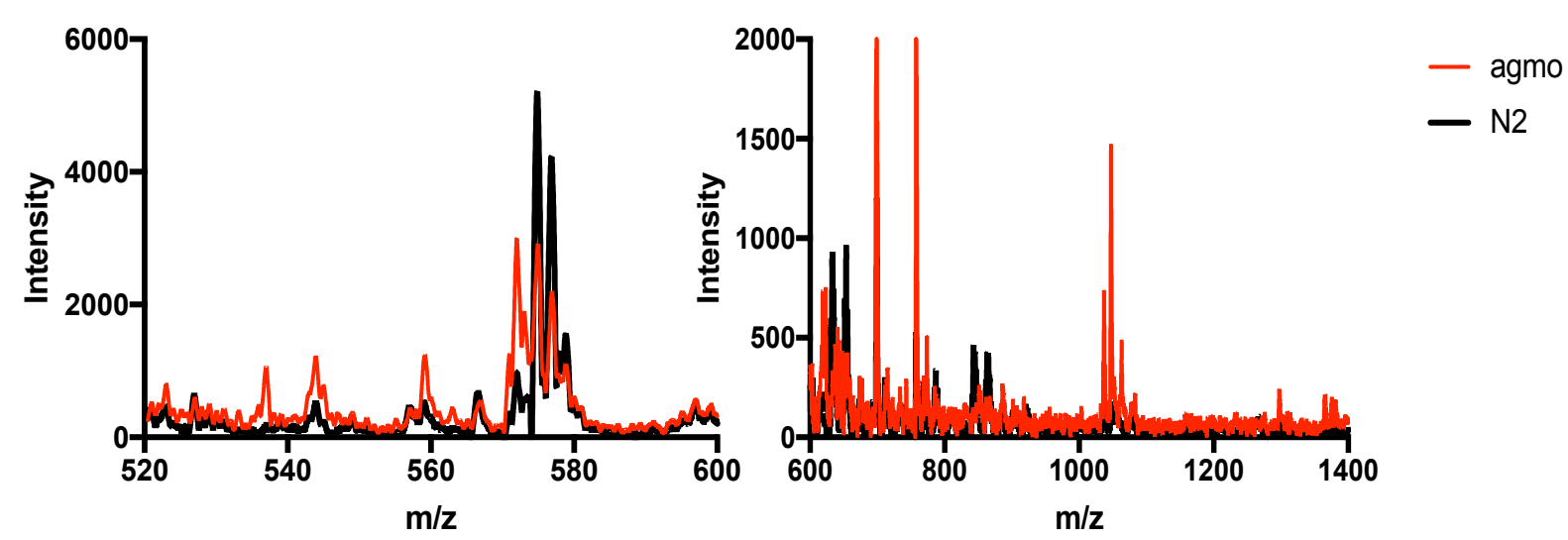

Figure 3 MALDI-MS spectra at different range exhibiting different peaks. In red the agmo-1 mutant and in dark the wild-type. Some differences are observed especially in the 600-1200 m/z region.

Lipid peaks were determined with an intensity above 500 (signal-to-noise ratio $>2$ ) and peaks were assigned (Table 3). Identification of lipids was performed using the LipidMAPS website ${ }^{39}$ with a mass tolerance of $\pm 0.1 \mathrm{~m} / \mathrm{z}$. All peaks were considered as possible ion adducts with potassium, sodium, with a possible loss of water and as singly and doubly charged, (MALDI primarily generates sodium and potassium adducts of singly or doubly charged species ${ }^{43}$ ). As expected, no ether lipids (coloured red in Table 3), were identified in the N2 wild-type strain, however up to two ether lipid species were identified in the agmo-1 mutant.

Some lipid species (rendered in green in Table 3) are observed in both the N2 and agmo-1 samples, including PA, PI, sulfoquinovosyldiacylglycerol (SQDG) and lyso-phosphatidylinositolmonophosphate (LPIP). In addition to these lipids, the N2 strain has some additional lipid types which include PS and MIPC. 
Table 3 The different lipids identified in SMA extracts using the LipidMAPS website ${ }^{39}$. In red the lipids which have been identified as ether-linked lipids (with O-indicating alkyl ether-linked lipids and P-indicating alkneyl-ether (plasmalogen) lipids) and in green lipids which are shared between the wild-type and mutant strain. In orange, the different ceramides with $d$ and $t$ designating the shorthand notation for sphingolipids and refer to 1,3-dihydroxy and 1,3,4-trihydroxy long-chain bases.

\begin{tabular}{|c|c|c|c|c|}
\hline \multicolumn{5}{|c|}{ agmo-1 } \\
\hline Mass (Da) & Abbreviation & Full name & Headgroup charge & Chain length and unsaturation \\
\hline 1539,587 & TG & Triradylglycerolpid & 0 & $99: 0$ \\
\hline 1385,993 & DGDG & Digalactosyldiacylglycerol & 0 & $65: 7$ \\
\hline 1369,024 & PIP & Glycerophosphoinositol monophosphate & $2-$ & $65: 1$ \\
\hline 1366,886 & $\mathrm{CL}$ or PIP & Cardiolipin or Glycerophosphoinositol monophosphate & $2-$ & $66: 7$ or $66: 8$ \\
\hline 1290,895 & PIP & Glycerophosphoinositol monophosphate & $2-$ & $60: 5$ \\
\hline 1279,758 & PIP & Glycerophosphoinositol monophosphate & $2-$ & $58: 7$ \\
\hline 1277,554 & $\mathrm{M}(\mathrm{IP})_{2} \mathrm{C}$ & Mannosyl-di-glycerophosphoinositol-ceramide & $2-$ & $\mathrm{d} 46: 3$ \\
\hline 1165,738 & SQDG & Sulfoquinovosyldiacylglycerol & $1-$ & $57: 9$ \\
\hline 1158,823 & $\mathrm{PE}$ & Glycerophosphoethanolamine & 0 & $64: 10$ \\
\hline 1148,848 & LacCer & Lactosyl ceramide & 0 & $\mathrm{t} 50: 0$ \\
\hline 1141,55 & $\mathrm{M}(\mathrm{IP})_{2} \mathrm{C}$ & Mannosyl-di-PI-ceramide & 2- & $\mathrm{d} 31: 2$ \\
\hline 1134,746 & PC & Glycerophosphocholine & 0 & $58: 12$ \\
\hline 1120,828 & PC or PE & Glycerophosphocholine or Glycerophosphoethanolamine & 0 & $58: 8$ or $61: 8$ \\
\hline 1107,787 & PS & Glycerophosphoserine & $1-$ & $60: 10$ \\
\hline 1106,841 & LacCer & Lactosyl ceramide & 0 & $\mathrm{~d} 50: 2$ \\
\hline 1087,602 & PIP & Glycerophosphoinositol monophosphate & $2-$ & $46: 8$ \\
\hline 1006,738 & DGDG & Digalactosyldiacylglycerol & 0 & $39: 1$ \\
\hline 947,71 & PA, PG or TG & Glycerophosphoglycerol & $1-$ & $0-53: 6, P-50: 6,58: 11$ \\
\hline 946,785 & TG & Triradylglycerolpids & 0 & $58: 9$ \\
\hline 888,844 & $\mathrm{DG}, \mathrm{TG}(\mathrm{O})$ or TG(P) & Diacylglycerol, Triacylglycerol & 0 & $54: 3,0-54: 3, P-54: 2$ \\
\hline 850,358 & \begin{tabular}{|c|}
$\mathrm{PIP}$ \\
\end{tabular} & Glycerophosphoinositol monophosphate & $2-$ & $27: 2$ \\
\hline 837,42 & SQDG & Sulfoquinovosyldiacylglycerols & $1-$ & $33: 5$ \\
\hline 824,388 & LPIP & Lyso-Glycerophosphoinositol monophosphate & $2-$ & $25: 1$ \\
\hline 811,422 & LPIP & Lyso-Glycerophosphoinositol monophosphate & $2-$ & $29: 3$ \\
\hline 760,465 & ShexCer & Sulfatides hexosyl ceramide & $1-$ & $\mathrm{d} 31: 1$ \\
\hline 751,324 & SQDG & Sulfoquinovosyldiacylglycerol & $1-$ & $27: 6$ \\
\hline 703,549 & HexCer & Hexosylceramide & 0 & $\mathrm{t} 32: 2$ \\
\hline 698,689 & CE or WE & Ceramide or Wax ester & 0 & $20: 0$ or $47: 5$ \\
\hline 655,376 & PA & Glycerophosphate & $1-$ & $30: 2$ \\
\hline 653,3 & SQDG & Sulfoquinovosyldiacylglycerol & $1-$ & $24: 6$ \\
\hline 603,446 & $\mathrm{PI}$ & Glycerophosphoinositol & $1-$ & $57: 0$ \\
\hline 576,466 & $P G$ & Glycerophosphoglycerol & $1-$ & $62: 4$ \\
\hline 566,379 & $\mathrm{PI}$ & Glycerophosphoinositol & $1-$ & $55: 9$ \\
\hline
\end{tabular}

\begin{tabular}{|c|c|c|c|c|}
\hline \multicolumn{5}{|c|}{ N2 } \\
\hline Mass (Da) & Abbreviation & Full name & Headgroup charge & Chain length and unsaturation \\
\hline 996,704 & PS & Glycerophosphoserine & $1-$ & $48: 1$ \\
\hline 811,422 & LPIP & Lyso-Glycerophosphoinositol monophosphate & 2- & 29:3 \\
\hline 760,332 & MIPC & Mannosyl-PI-ceramide & 1- & $\mathrm{t} 18: 0$ \\
\hline 655,376 & PA & Glycerophosphate & 1- & $30: 2$ \\
\hline 653,3 & SQDG & Sulfoquinovosyldiacylglycerol & 1- & 24:6 \\
\hline 566,379 & PI & Glycerophosphoinositol & 1- & $55: 9$ \\
\hline
\end{tabular}




\section{Discussion}

The SMA lipid extraction method used here, appears to be milder than protocols involving detergent or organic extractions ${ }^{6}$, and the fact that $95 \%$ of the nematodes are viable after treatment with SMA, suggests that the integrity of the cell membranes in the $C$. elegans body is not severely compromised, and that the polymer primarily extracts lipids from the cuticle.

The DLS data suggest that the lipid extraction from the worms by SMA, results in the formation of Lipodisq nanoparticles, with diameters measured in the range $6 \mathrm{~nm}-25 \mathrm{~nm}$ (Table 1), similar to those previously reported $22-24,44,45$. The sizes of the particles formed, differs between the N2 and agmo-1 strains, consistent with a difference in lipid content and also might indicate differences in protein content. Additionally, the N2 cuticle is more resistant to SMA treatment, in agreement with earlier studies which indicate that the agmo-1 cuticle is more fragile ${ }^{9}$. The accumulation of ether lipids may compromise the stability of the agmo-1 cuticle, despite its ability to prevent Verde1 attachment to C. elegans surface.

Identification of lipid headgroups was carried by TLC and some retention factors measured in previous publications ${ }^{6}$ are similar to our data and can be identified ${ }^{46}$. Therefore, the $R_{f}=0.24$ could be LPC-type lipids and the $R_{f}=0.61$, could be PG-type lipids. Some of the lipids found in the Lipodisq extraction were also identified by Blaxter and colleagues, such as PE and ceramides, however we were unable to identify PC or PI from in our data (Table 2). The presence of SQDG and ceramide-type lipids (Figure 3 and Table 3 ) is consistent with literature, especially for the sulfonated lipids, which are responsible for the negative charge on the cuticle. All of the lipids identified by the TLC analysis are lipid species expected from previous genetic studies of $C$. elegans ${ }^{16}$.

Since the agmo-1 mutant lacks alkylglycerol monooxygenase (AGMO), which cleaves the ether bond of alkylglycerols, we expect to observe some differences in the abundance of ether-linked lipids in the mutant. Compared to the wild-type strain, more lipids were observed and identified for the agmo-1 mutant, as seen by the TLC (Figure 3). The lipids highlighted (in red in Table 3) are ether-linked lipid species, which have been speculated to accumulate at the cuticle surface due to the lack of the AGMO enzyme. The literature suggest that sulfatides, PE and PI have the 
lowest detectability threshold ${ }^{47}$. Therefore their identification in this experiment, may mean that the sample contains a high amount of these lipids, which is not surprising as PI and sulfatides lipids have been suggested to regulate the cuticle surface charge ${ }^{6}$.

The accumulation of some ether-linked lipids at the cuticle surface of the mutant, may prevent bacterial adherence by masking binding targets on the worm surface. The agmo-1 nematodes float on aqueous buffer (unlike wild-type worms) consistent with an increased abundance of ether-linked lipids and waxes, both of which have been reported to affect buoyancy ${ }^{48}$. The wildtype cuticle also contains greater relative abundance of negatively charged lipid headgroups, which are diluted by the ether-linked lipids and ceramides in the agmo-1 mutant, which may also explain the differences in the apparent ease of solubilisation of the cuticle lipids by the negatively charged SMA polymer.

Surprisingly we also observe some ester-linked lipid species in the agmo-1 mutant which are not found in the N2 strain. We propose that a compensation mechanism is effected, by which results in the upregulation of other lipid synthesis pathways and the more heterogeneous lipid composition of the mutant cuticle. A similar phenomenon has been observed in murine macrophage-like cells, in which a decrease in AGMO activity (and increase in free alkylglycerol lipid types) resulted in widespread changes in the cell lipidome, including changes in the abundance of glycosylated ceramides and cardiolipin ${ }^{10}$. In our case, we found several glucosylceramides in the agmo-1 mutant, which have been suggested to be located in the nematode's cuticle $^{49}$, some tri- and diacylglycerols and only one cardiolipin ${ }^{10}$. We suggest that the protein composition also differs as a result of changes in lipid-protein interactions.

Ether lipid-deficient strains in C. elegans accumulate high levels of stearic acid (18:0) in several types of ester-linked lipid, presumably to compensate for the reduced rigidity that would be conferred by the ether lipids ${ }^{50}$. It is striking that longer chain length lipids with high molecular weights are observed in the agmo-1 mutant but not the N2 strain. The wild-type cuticle has a less diverse lipidome, and the lipids present generally have shorter acyl chain lengths than the mutant. 


\section{Conclusion}

We have demonstrated that lipid extraction by SMA may be used to study the lipidome of the C. elegans cuticle. We have shown that SMA can be used on living organisms without affecting their viability, compared to harsher lipid extraction methods such as those requiring organic solvents (Figure 1). We have identified an accumulation of ether-linked lipids in the agmo-1 mutant nematode cuticle, which is consistent with the deletion of a functional alkylglycerol monooxygenase (AGMO) in this strain ${ }^{9}$. The presence and abundance of other lipid species also differ between the wild-type N2 and agmo-1 worms, suggesting that the mutant strain attempts to compensate for the increase in ether-linked lipids by modulating other lipidsynthesis pathways. Together these differences not only affect the fragility of the cuticle ${ }^{9}$ and the buoyancy of the worm in aqueous buffer, but also the interactions between the exoskeleton and surface-adhering bacteria ${ }^{9}$.

\section{Acknowledgments}

We thank Dr Holger Kramer for access to MS equipment and Peter Fisher for technical assistance. This study was partly funded by MRC grant MR/J001309/1 and a Faucett Catalyst Grant (to JH). We also thank Malvern Cosmeceutics (Steve Tonge and Andy Harper) for their continued support and supply of SMA polymer. 
References

1. J. Sulston and J. Hodgkin. The Nematode Caenorhabditis elegans. (Cold Spring Harbor Laboratory, 1988).

2. Brenner, S. The genetics of Caenorhabditis elegans. Genetics (1974). doi:10.1002/cbic. 200300625

3. Bloom, F. E. Staying afloat on the seas of data. Science (80-. ). 282, 1989 (1998).

4. Riddle, D. L., Blumenthal, T. \& Meyer, B. J. C. elegans. (Cold Spring Harbor, 1997).

5. $\quad$ Page, A. The cuticle. WormBook 1-15 (2007). doi:10.1895/wormbook.1.138.1

6. Blaxter, M. L. Cuticle surface proteins of wild type and mutant Caenorhabditis elegans. J. Biol. Chem. 268, 6600-9 (1993).

7. Hodgkin, J., Félix, M.-A., Clark, L. C., Stroud, D. \& Gravato-Nobre, M. J. Two Leucobacter Strains Exert Complementary Virulence on Caenorhabditis Including Death by WormStar Formation. Curr. Biol. 23, 2157-2161 (2013).

8. Watschinger, K. \& Werner, E. R. Alkylglycerol monooxygenase. IUBMB Life 65, 366-372 (2013).

9. Loer, C. M. et al. Cuticle integrity and biogenic amine synthesis in Caenorhabditis elegans require the cofactor tetrahydrobiopterin (BH4). Genetics 200, 237-253 (2015).

10. Watschinger, K. et al. Tetrahydrobiopterin and alkylglycerol monooxygenase substantially alter the murine macrophage lipidome. Proc. Natl. Acad. Sci. 112, 24312436 (2015).

11. Gravato-Nobre, M. J. et al. Multiple genes affect sensitivity of Caenorhabditis elegans to the bacterial pathogen Microbacterium nematophilum. Genetics 171, 1033-1045 (2005).

12. Chisholm, A. D. \& Xu, S. The Caenorhabditis elegansepidermis as a model skin. II: differentiation and physiological roles. Wiley Interdiscip. Rev. Dev. Biol. 1, 879-902 (2012).

13. Johnstone, I. L. The cuticle of the nematode Caenorhabditis elegans: A complex collagen structure. BioEssays 16, 171-178 (1994).

14. Gounaris, K., Smith, V. P. \& Selkirk, M. E. Structural organisation and lipid composition of the epicuticular accessory layer of infective larvae of Trichinella spiralis. Biochim. Biophys. Acta - Biomembr. 1281, 91-100 (1996).

15. L, P., Kusel J R, S. H. V \& W., K. M. Biophysical properties of the nematode surface. Parasit. nematodes---Membranes, antigens genes (1991).

16. Witting, M. \& Schmitt-Kopplin, P. The Caenorhabditis elegans lipidome: A primer for lipid analysis in Caenorhabditis elegans. Arch. Biochem. Biophys. 589, 27-37 (2016).

17. Lee, D. L. The biology of the nematodes. (CRC Press, 2002).

18. Matyash, V., Liebisch, G., Kurzchalia, T. V., Shevchenko, A. \& Schwudke, D. Lipid extraction by methyl- tert -butyl ether for high-throughput lipidomics. J. Lipid Res. 49, 1137-1146 (2008).

19. Zhang, R. \& Hou, A. Host-Microbe Interactions in Caenorhabditis elegans. ISRN Microbiol. 2013, 1-7 (2013).

20. Shi, S., Luke, C. J., Miedel, M. T., Silverman, G. A. \& Kleyman, T. R. Activation of the caenorhabditis elegans degenerin channel by shear stress requires the MEC-10 subunit. J. Biol. Chem. 291, 14012-14022 (2016).

21. Scheidelaar, S. et al. Molecular Model for the solubilization of membranes into nanodisks by styrene maleic acid copolymers. Biophys. J. 108, 279-290 (2015). 
22. Dörr, J. M. et al. The styrene-maleic acid copolymer: a versatile tool in membrane research. Eur. Biophys. J. 45, 3-21 (2016).

23. Esmaili, M. \& Overduin, M. Membrane biology visualized in nanometer-sized discs formed by styrene maleic acid polymers. Biochim. Biophys. Acta-Biomembr. 1860, 257-263 (2018).

24. Lee, S. C. et al. A method for detergent-free isolation of membrane proteins in their local lipid environment. Nat. Protoc. 11, 1149-1162 (2016).

25. Dominguez Pardo, J. J. et al. Solubilization of lipids and lipid phases by the styrenemaleic acid copolymer. Eur. Biophys. J. 46, 91-101 (2017).

26. Parmar, M. et al. Using a SMALP platform to determine a sub-nm single particle cryoEM membrane protein structure. Biochim. Biophys. Acta-Biomembr. 1860, 378-383 (2018).

27. Sun, C. et al. Structure of the alternative complex III in a supercomplex with cytochrome oxidase. Nature 557, 123-126 (2018).

28. Broecker, J., Eger, B. T. \& Ernst, O. P. Crystallogenesis of Membrane Proteins Mediated by Polymer-Bounded Lipid Nanodiscs. Structure 25, 384-392 (2017).

29. Dörr, J. M. et al. Detergent-free isolation, characterization, and functional reconstitution of a tetrameric $\mathrm{K}^{+}$channel: The power of native nanodiscs. Proc. Natl. Acad. Sci. 111, 18607-18612 (2014).

30. Jamshad, M. et al. G-protein coupled receptor solubilization and purification for biophysical analysis and functional studies, in the total absence of detergent. Biosci. Rep. 35, 1-10 (2015).

31. Logez, C. et al. Detergent-free Isolation of Functional G Protein-Coupled Receptors into Nanometric Lipid Particles. Biochemistry 55, 38-48 (2016).

32. Gulati, S. et al. Detergent-free purification of ABC (ATP-binding-cassette) transporters. Biochem. J. 461, 269-278 (2014).

33. Postis, V., Rawson, S., Mitchell, J., ... S. L.-... et B. A. (BBA \& 2015, undefined. the Use of Sma Lipid Particles As a Novel Membrane Protein Scaffold for Structure Study By Negative Stain Em.Pdf. Elsevier 1848, 496-501 (2015).

34. Swainsbury, D. J. K. et al. Probing the local lipid environment of the Rhodobacter sphaeroides cytochrome bc1and Synechocystis sp. PCC 6803 cytochrome b6f complexes with styrene maleic acid. Biochim. Biophys. Acta - Bioenerg. 1859, 215-225 (2018).

35. Long, A. R. et al. A detergent-free strategy for the reconstitution of active enzyme complexes from native biological membranes into nanoscale discs. BMC Biotechnol. 13, 41 (2013).

36. Reading, E. Structural Mass Spectrometry of Membrane Proteins within Their Native Lipid Environments. Chem. - A Eur. J. (2018). doi:10.1002/chem.201801556

37. Reading, E. et al. Interrogating Membrane Protein Conformational Dynamics within Native Lipid Compositions. Angew. Chemie - Int. Ed. 56, 15654-15657 (2017).

38. Bligh, E. . \& Dyer, W. . Canadian Journal of Biochemistry and Physiology. J. Biochem. Physiol. 37, 911-917 (1959).

39. Sud, M. et al. LMSD: LIPID MAPS structure database. Nucleic Acids Res. 35, 527-532 (2007).

40. Pappas, A. Epidermal surface lipids. Dermatoendocrinol. 1, 72-76 (2009).

41. Bouwstra, J. A. et al. Role of ceramide 1 in the molecular organization of the stratum corneum lipids. J. Lipid Res. 39, 186-196 (1998). 
42. Hou, N. S. \& Taubert, S. Function and regulation of lipid biology in Caenorhabditis elegans aging. Front. Physiol. 3 MAY, 1-10 (2012).

43. Abonnenc, M., Qiao, L., Liu, B. \& Girault, H. H. Electrochemical Aspects of Electrospray and Laser Desorption/Ionization for Mass Spectrometry. Annu. Rev. Anal. Chem. 3, 231-254 (2010).

44. Orwick, M. C. et al. Detergent-free formation and physicochemical characterization of nanosized lipid-polymer complexes: Lipodisq. Angew. Chemie - Int. Ed. 51, 4653-4657 (2012).

45. Orwick-Rydmark, M. et al. Detergent-free incorporation of a seven-transmembrane receptor protein into nanosized bilayer lipodisq particles for functional and biophysical studies. Nano Lett. 12, 4687-4692 (2012).

46. TLC solvent systems. Available at: https://avantilipids.com/tech-support/analyticalprocedures/tlc-solvent-systems/.

47. Fuchs, B., Süß, R. \& Schiller, J. An update of MALDI-TOF mass spectrometry in lipid research. Prog. Lipid Res. 49, 450-475 (2010).

48. Magnusson, C. D. \& Haraldsson, G. G. Ether lipids. Chem. Phys. Lipids 164, 315-340 (2011).

49. Kage-Nakadai, E. et al. Two very long chain fatty acid acyl-CoA synthetase genes, acs-20 and acs-22, have roles in the cuticle surface barrier in Caenorhabditis elegans. PLoS One 5, (2010).

50. Watts, J. L. \& Ristow, M. Lipid and carbohydrate metabolism in Caenorhabditis elegans. Genetics 207, 413-446 (2017). 


\section{Supplementary information}

6. Material and methods

\subsection{SMA polymer hydrolysis}

Styrene-maleic anhydride polymer (SMAnh), in a molar ratio of styrene to maleic anhydride of 3:1, was kindly provided by Malvern Cosmeceutics (Great Malvern, UK). The polymer was hydrolysed using a $1 \mathrm{M} \mathrm{NaOH}$ solution ( $5 \% \mathrm{w} / \mathrm{v}$ final) (Fisher) at $80-90^{\circ} \mathrm{C}$ for $1-2 \mathrm{~h}$, during which the solution changed from cloudy to a transparent yellow colour. $5 \mathrm{M} \mathrm{HCl}$ was then added to precipitate the SMA polymer, which was pelleted by centrifugation (2000g, 5 min, RT).

The pellet was re-solubilised in water and was subjected to multiple washing and centrifugation steps (2000g, 5 minutes). Once washed, double distilled water $\left(\mathrm{ddH}_{2} \mathrm{O}\right)$ was added to the solution and dialysis was performed overnight in order to remove the excess of salt and to adjust the $\mathrm{pH}$. A transparent yellowish SMA solution resulted, which was lyophilized to yield a white powder. $\mathrm{ddH}_{2} \mathrm{O}$ water or buffer was added to a final concentration of $125 \mathrm{mg} / \mathrm{ml}$ and the $\mathrm{pH}$ was adjusted to $\mathrm{pH} 8$.

\subsection{Thin layer chromatography}

The lipids were spotted onto a TLC silica gel (Merck). Plates were developed with a methanol/chloroform/ammonium hydroxide (65:25:4 v/v/v) solvent mixture [280]. Lipid bands (Fig. SI.1) were visualized by spraying the plates with molybdenum blue (Sigma) and charred at $200^{\circ} \mathrm{C}$. Retention factors $(\mathrm{Rf})$ were calculated. 


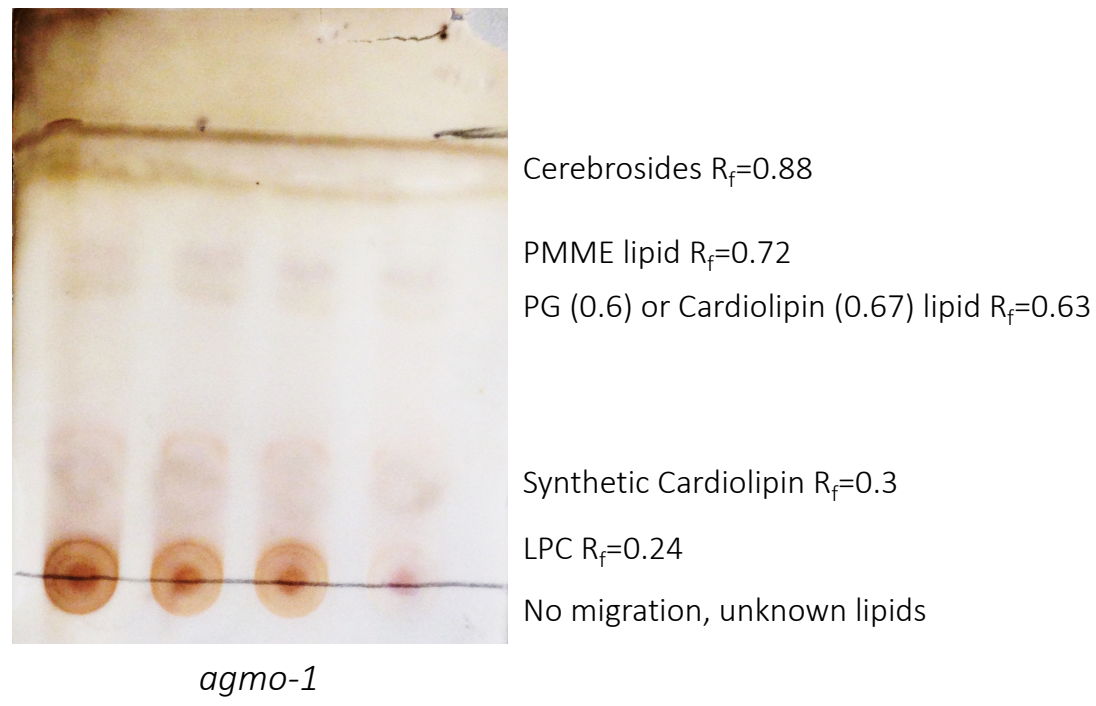

Figure 1. SI. Thin layer chromatography experiments. The agmo-1 lipid extraction is shown here where different concentrations of lipids were spotted on the plate. Lipid headgroups were identified by their retention factors. 